\title{
Is Management of Asthma is different in Pregnancy?
}

\section{A A Mamun $\mathrm{SM}^{1 *}$, Shaheed $\mathrm{S}^{2}$ and Sarker $\mathrm{S}^{3}$}

${ }^{1}$ Senior Consultant \& coordinator of Respiratory Medicine, Square Hospitals, Bangladesh

${ }^{2}$ Associate Professor of Gynaecology \& Obstetrics, Dhaka Central international Medical College, Bangladesh

${ }^{3}$ Specialist, Respiratory Medicine, Square Hospitals, Bangladesh

*Corresponding author: SM A A Mamun, Senior Consultant \& coordinator of Respiratory

Medicine, Square Hospitals, Bangladesh, Email: mamundr69@gmail.com

\section{Review Article}

Volume 6 Issue 1

Received Date: April 26, 2021

Published Date: May 11, 2021

DOI: $10.23880 /$ oajprs-16000139

\section{Abstract}

Bronchial asthma \& its exacerbations is not uncommon during pregnancy and its prevalence is increasing in the community. Exacerbations are a major clinical problem during pregnancy with $>40 \%$ of women necessitates medical help \& if not managed properly, results poor outcomes for mothers and their babies, including low birth weight and preterm delivery. The effective asthma management during pregnancy needs an good action plan to maintain the best possible asthma control. This may achieved by planning of day and night-time symptoms control and maintain lung function to achieve good $\mathrm{O}_{2}$ saturation 24 hours. This is the key approach to maintain fetal oxygenation homogeneous throughout the pregnancy. Different guidelines recommend that physicians should provide asthma advice and review patients with relevant investigation prior to coceive and then managing asthma actively during pregnancy, with regular 4-weekly review, provision of a written action plan, use of preventer medications as indicated for other adults with asthma, and management of co-morbid conditions if any.

Keywords: Bronchial Asthma; Pregnancy; Inhaler Medication

\section{Introduction}

Bronchial asthma is one of the most common chronic medical conditions encountered during pregnancy, occurring in 3 to 8 percent of pregnant women [1,2]. Pregnancy may be associated with changes in the course of asthma and asthma may affect the outcome of pregnancy. Bronchial asthma is fairly affecting general population, including some women who have never had it before their pregnancy. During the period of asthma not only affects patients, but it can also affect the fetus in the utero as it gets $\mathrm{O} 2$ from mother. But this does not mean that having asthma will make pregnancy more difficult or dangerous to mother or fetus. Pregnant women who have bronchial asthma that is properly controlled generally have normal pregnancies with little or no increased risk to themselves or their developing babies $[3,4]$.

Most of the asthma medication are safe to use for a pregnant woman. After years of research, experts now say that it is far safer to manage asthma with medicine than it is to leave asthma untreated during pregnancy.

\section{Risks of Uncontrolled Asthma to Pregnant Women}

Usually women not previously had asthma, may not think that shortness of breath or wheezing during her pregnancy is asthma. If she knows that she has asthma, but may not consider it a concern if she only has mild symptoms. But asthma can affect seriously both mother and fetus and you 


\section{Open Access Journal of Pulmonary \& Respiratory Sciences}

should act accordingly. Some women experienced shortness of breath or/and wheezing $1^{\text {st }}$ time during pregnancy but some experienced severe attack of asthma, who has previous asthma in control.

\section{If Asthma is not Controlled}

\section{Risks of women's health include:}

1. High blood pressure during the pregnancy.

2. Pre-eclampsia a condition that causes high blood pressure and can affect the placenta, kidneys, liver and brain.

\section{Risks to the fetus include:}

1. Abnormally slow growth of the fetus (intrauterine growth retardation). When born, the baby appears small.

2. Birth before the $37^{\text {th }}$ week of pregnancy (preterm birth).

3. Low birth weight.

4. Death immediately before or after birth (perinatal mortality)

So good control of asthma is needed to ensure healthy outcome of mother and fetus (Table 1).

\begin{tabular}{|l|c|c|c|}
\hline \multicolumn{1}{|c|}{ Variable } & Well Controlled Asthma & Asthma not in good Control & Poor Controlled Asthma \\
\hline 1. Frequency of symptoms & $<$ days/week & $>2$ days/week & Throughout the week \\
\hline 2. Frequency of night times awakening & $<$ days/month & $1-3$ times/week & $>4$ days/week \\
\hline 3. Interference with normal activity & None & Some & Extreme \\
\hline 4.FEV1 or PEFR & $>80$ & $60-80 \%$ & $<60 \%$ \\
\hline 5. Exacerbation need systemic steroid & $0-1$ in past 12 month & $>2 / 12$ month & $>2 / 12$ month \\
\hline
\end{tabular}

Table 1: Assesment of asthma control in pregnant women.

\section{Pulmonary Function Changes in Pregnancy}

Most of the physiological parameters of lung functions are altered in pregnancy. There is an increase in oxygen consumption of $20 \%$ with an increased maternal metabolic rate of $15 \%$ [5]. The functional residual capacity (amount of air remaining in the lungs during normal breathing) decreases by $17-20 \%$; the residual volume (air remaining in the lungs after maximal expiration) decreases by 20 25\%; the tidal volume (air displaced between inhalation and exhalation with no extra effort) increases by $30-50 \%$; the expiratory reserve volume (the air that can be further pushed out at the end of normal expiration) decreases by 5-15\%; and the minute volume/ventilation (volume of air inhaled or exhaled in 1 minute) increases by $30-50 \%$ [5-7].

The respiratory rate, $\mathrm{FEV}_{1}$ and peak expiratory flow rate (PEFR) are unchanged. This makes the latter two very suitable methods for assessing pulmonary function in pregnancy [6,7]. The changed minute ventilation and increased tidal volume results in a relative hyperventilation with a subsequent respiratory alkalosis (pH of 7.40 to 7.45; $\mathrm{PCO}_{2}$ of 28 to $32 \mathrm{mmHg}$ ) compensated for by renal excretion of bicarbonate. There is a mild increase in maternal arterial $\mathrm{PO}_{2}$ (106-110 mmHg) with the umbilical venous $\mathrm{PO}_{2}$ being lower than that of the placental venous channels. As such any maternal hypoxia quickly results in substantial fetal hypoxia. Coleman and Rund reported that a $\mathrm{PO}_{2}$ of 60 causes fetal jeopardy. The length of the hypoxia would relate to the degree of fetal compromise.

\section{Asthma Treatment and Pregnancy}

Virtually there is no difference between the management of bronchial asthma in pregnant women and non-pregnant women. Like all people with asthma, pregnant women need to have an asthma action plan to help them control inflammation and prevent and control asthma attacks \& maintain record.

Although there is a general concern about any medication use in pregnancy the advantage of actively treating asthma in pregnancy markedly outweigh any potential risks of usual controller and reliever medications. For this reason, using medications to achieve good symptom control and prevent exacerbations is justified even when their safety in pregnancy has not been unequivocally proven. Use of ICS, beta- 2 agonist, montelukast or theophylline is not associated with an increased incidence of fetal abnormalities [8]. Importantly, ICS reduce the risk of exacerbations of asthma during pregnancy. And cessation of ICS during pregnancy is a significant risk factor for exacerbations [8].

The current approach to management seeks to achieve optimal disease control, which means using medication and other management strategies to achieve minimal symptoms (ideally none) of asthma, optimal lung function, and to reduce the future risks of asthma exacerbations [8].

Pregnant women should note, whether fetal kicks decrease over time. If she notices less fetal activity during 


\section{Open Access Journal of Pulmonary \& Respiratory Sciences}

an asthma attack, contact with doctor or emergency immediately to get instructions.

Things to think about for asthma in pregnant women include the following: If more than one health professional is involved in the pregnancy and asthma care, they must communicate with each other about treatment. The obstetricians must be involved with asthma care. According to the current recommendation $s$ regular monitoring of asthma monthly during pregnancy is recommended for all pregnant women with asthma [8].

Some women with asthma stop or reduce their use of asthma controller medication in early pregnancy [9], often without consulting their doctor, and reduced adherence with controller medication during pregnancy is associated with increased risk of asthma -related emergency department visits. The reason for abstaining controller medication is very likely to be fear of harmful effects of the drugs on the foetus [10]. Non-adherence to controller medication is, in general, a major risk factor for asthma exacerbations and the risk of having an exacerbation is reduced by more than $75 \%$ among women who are using controller medication regularly. Asthma exacerbation's during the first trimester of pregnancy increase the risk of congenital malformation [11].

Monitor lung function carefully throughout your pregnancy to ensure that your growing fetus gets enough oxygen. Because asthma severity changes for about twothirds of patients during pregnancy, should have monthly check ups with her doctor to monitor symptoms and lung function. Doctor will use either spirometry or a peak flow meter to measure lung function. Monitor fetal movements daily after 28 weeks [12].

If asthma is not well controlled or if patients have moderate or severe asthma, think about having ultrasound after 32 weeks to monitor fetal growth. Ultrasound exams can also help to check on the fetus after an asthma attack [12].

All patients should be educated regarding the relationship between asthma and pregnancy, and they should be taught about self-treatment, including inhaler techniques, adherence to medication, and control of potential environmental factors.

It is well -documented that active and passive tobacco smoke exposure is a major risk factor for worse asthma control, excessive decline in FEV 1, and impaired responses to ICS and oral steroids. Asthmatic women who are current smokers during pregnancy have more severe asthma exacerbations during pregnancy relative to women who are non -smokers [13].
Try to do more to avoid and control asthma triggers (such as tobacco smoke or house dust mites, pollen), so that you can take less medicine if possible. Many women have nasal symptoms, and there may be a link between increased nasal symptoms and asthma attack. Well control of nasal symptoms by applying safe nasal preparation (Budesonide nasal spray preferable), gastro esophageal reflux disorder (GERD), which is common in pregnancy, may also cause symptoms.

The appropriate management of common coexisting conditions that can aggravate asthma, such as rhinitis, sinusitis, and gastro esophageal reflux, can improve asthma control. Women who smoke must be informed of the potential adverse effects of smoking on the fetus, which may add to the fetal effects of uncontrolled asthma and should be strongly encouraged to quit. Advice on environmental control measures for reducing exposure to allergens can be provided on the basis of the results of allergy testing.

\section{Medications for Asthma}

Medications for asthma are divided into long-term controller medications that prevent the manifestations of asthma (inhaled corticosteroids, long-acting $\beta$ - agonists, leukotriene modifiers, cromolyn, and theophylline) and rescue therapy that provides quick relief of symptoms (primarily short-acting inhaled $\beta$-agonists) [14-15].

In pregnancy, LABA are considered for use in moderate to severe asthma, in combination with ICS therapy. Few studies have examined their safety in this context, most being post marketing surveillance studies or small cohort or casecontrol studies examining congenital malformations. One study reported no adverse outcomes among 65 pregnant women who used salmeterol [15].

A recent study from Quebec, found no significantly increased odds of any or major malformations with first trimester LABA use (adjusted OR 1.37, 95\% CI 0.92, 2.17 for any malformations) [16]. There was an increased risk of major cardiac malformations (adjusted OR 2.38, 95\% CI) among asthmatic users of LABA compared to non-users of LABA in another small group study [17]. Based on a longer period of availability, 2004 guidelines recommended salmeterol as the preferred LABA used in pregnancy [18].

Evidence of the efficacy of these drugs during pregnancy is largely extrapolated from studies involving patients who were not pregnant. To our knowledge, only two randomized, controlled trials specifically involving pregnant patients with asthma have been conducted. These studies showed that inhaled beclomethasone is more effective than theophylline in improving pulmonary function [19] in other 


\section{Open Access Journal of Pulmonary \& Respiratory Sciences}

studies. Theophylline was as effective as beclomethasone diproprianate but was associated with a higher side effect rate and higher discontinuation rates [20].

On balance, given the evidence in pregnancy and infancy for adverse outcomes from exacerbations during pregnancy including due to lack of ICS or poor adherence, and evidence for safety of usual doses of ICS and LABA and a low priority should be placed on stepping down treatment (however guided) until after delivery. and ICS should not be stopped in preparation for pregnancy or during pregnancy [8].

Neonatal hypoglycemia may be seen, especially in preterm babies, when high doses of Beta-agonists have been given within the last 48 hours prior to delivery. If high doses of SABA have been given during and before delivery, blood glucose levels should be monitored in the baby (especially if preterm) for the first 24 hours [8].
Oral corticosteroids: During the first trimester may have a slight increased risk of isolated cleft lip, with or without cleft palate, $(0.3 \%$ versus $0.1 \%)$. There are also associations with preterm delivery, low birth weight and preeclampsia [21]. It is however, difficult to delineate what is an attributable effect from the corticosteroids versus the effects of severe or uncontrolled asthma.

Inhaled corticosteroids reduces risk of asthma exacerbations in pregnancy and improved lung function $\left(\mathrm{FEV}_{1}\right)$ [1]. There are no data showing any increase in congenital malformations or adverse perinatal outcomes [22]. The majority of experience with ICS to date is with budesonide. The numbers of women taking Leukotriene inhibitor notably montelukast having no significant adverse effect are small with minimal data available [23] (Table 2).

\begin{tabular}{|c|c|c|}
\hline Steps & Preferred Controller Medication & Alternate Controller Medication \\
\hline 1 & None & None \\
\hline 2 & Low dose inhaled corticosteroid & LTRA, Theophylline, Cromolyn \\
\hline 3 & Medium dose inhaled corticosteroid & $\begin{array}{c}\text { Low dose inhaled corticosteroid + LTRA or LABA or } \\
\text { Theophylline }\end{array}$ \\
\hline 4 & Medium dose inhaled corticosteroid +LABA & $\begin{array}{c}\text { Medium dose inhaled corticosteroid+ LRTA or } \\
\text { Theophylline }\end{array}$ \\
\hline 5 & High dose inhaled corticosteroids +LABA & None \\
\hline 6 & High dose inhaled corticosteroids +LABA+ oral \\
corticosteroids & None \\
\hline
\end{tabular}

Table 2: Medication for asthma control in pregnant women.

\section{Is the Flu Shot during Pregnancy Safe?}

Yes, the flu shot during pregnancy is not only safe, it's a must. The seasonal flu shot has been given safely to millions of pregnant women over many years and has not been shown to cause harm to expecting moms or their babies. Getting an influenza (flu) vaccine is the first and most important step in protecting against flu. Pregnant women should get a flu shot and not the nasal spray flu vaccine. Flu shots given during pregnancy help protect both the mother and her baby from flu. Vaccination has been shown to reduce the risk of fluassociated acute respiratory infection in pregnant women by up to one-half $[24,25]$.

There is good evidence that pregnant women have a higher chance of developing complications if they get flu, particularly in the later stages of pregnancy. One of the most common complications of flu is bronchitis a chest infection that can become serious and develop into pneumonia.

\section{Conclusions and Recommendations}

Although women with well-controlled asthma in pregnancy generally have good pregnancy outcomes. But poor controlled asthma may increase the risk of adverse perinatal outcomes. This patient should be educated regarding the potential risks of uncontrolled asthma for herself and her pregnancy. We would recommend good allergens control of food and nonfood (mites \& pollen) variety. Use of inhaled budesonide $(180 \mu \mathrm{g}$ per puff, two puffs twice a day) over other inhaled corticosteroids are recommended because more safety data are available on the use of this drug during the gestational period.

The patient should also be instructed to use the inhaler properly after right demonstration, and she should be given a personalized self-treatment action plan for asthma that includes instructions regarding the maintenance medication schedule, doses of rescue therapy for increased symptoms, 


\section{Open Access Journal of Pulmonary \& Respiratory Sciences}

and when and how to seek urgent or emergency care. We would recommend follow-up every 1 to 2 weeks initially to ensure that asthma control is achieved and after then at least monthly throughout the pregnancy. This Review has some limitation of lacking enough research studies on this topic. Our further effort will be targeting more relevant and current research studies.

\section{References}

1. Van Zutphen AR, Bell EM, Browne ML, Lin S, Lin AE, et al. (2015) Maternal asthmamedication use during pregnancy \& risk of congenital heart defects. Birth defects Res A ClinMol Teratol 103(11): 951-961.

2. Namazy J, Schatz M (2016) The treatment of Allergic Respiratory Disease During Pregnancy. J Investig Allergol Clin Immunol 26(1): 1-7.

3. Schatz M, Dombrowski MP (2009) Asthma in Pregnancy. N Engl J Med 360: 1862-1869.

4. Namazy JA, Schatz M (2005) Pregnancy and asthma: recent developments. Curr Opin Pulm Med 11(1): 56-60.

5. Hardy-Fairbanks A, Baker ER (2010) Asthma in pregnancy: pathophysiology, diagnosis and management. Obstet Gynecol Clin N Am 37(2): 159-172.

6. Jensen D, Duffin J, Lam YM, Webb KA, Simpson JA, et al. (2008) Physiological mechanisms of hyperventilation during human pregnancy. Respir Physiol Neurobiol 161(1): 76-86.

7. Kolarzyk E, Szot WM, Lyszczarz J (2005) Lung function and breathing regulation parameters during pregnancy. Arch Gynecol Obstet 272(1): 53-58.

8. GINA Report (2021) Global Strategy for Asthma Management and Prevention.

9. Enriquez R, Wu P, Griffin MR, Gebretsadik T, Shintani A, et al. (2006) Cessation of asthma medication in early pregnancy. American journal of obstetrics and gynecology 195(1): 149-153.

10. Matsui D (2012) Adherence with drug therapy in pregnancy. Obstetrics and gynecology international 796590.

11. Blais L, Kettani FZ, Forget A, Beauchesne MF, Lemiere C (2015) Asthma exacerbations during the first trimester of pregnancy and congenital malformations: revisiting the association in a large representative cohort. Thorax 70(7): 647-652.

12. National Asthma Education and Prevention Program
(2005) Working Group Report on Managing Asthma During Pregnancy: Recommendations for Pharmacologic Treatment.

13. Stapleton M, Howard-Thompson A, George C, Hoover RM, Self TH (2011) Smoking and asthma. J Am Board Fam Med 24(3): 313-322.

14. Kallen B, Olausson PO (2007) Use of anti-asthmatic drugs during pregnancy. 3. Congenital malformations in the infants. Eur J Clin Pharmacol 63(4): 383-388.

15. Mann RD, Kubota K, Pearce G, Wilton L (1996) Salmetrol: a study by prescription-event monitoring in a UK cohort of 15,407 patients. J Clin Epidem 49(2): 247-250.

16. Eltonsy S, Forget A, Blias L (2011) Beta 2 -agonists use during pregnancy and the risk of congenital malformations. Birth Defects Res 91(11): 937-947.

17. Clifton VL, Engel P, Smith R, Gibson P, Brinsmead $M$, et al. (2009) Maternal and neonatal outcomes of pregnancies complicated by asthma in an Australian population. Aust NZ J Obstet Gynaecol 49(6): 619-626.

18. Busse W (2004) NAEPP Expert Panel report. Managing asthma during pregnancy: Recommendations for pharmacologic treatment-2004 Update. J Allergy Clin Immunol 115(1): 34-46.

19. Mitchell PD, Schatz M, Thom EA, Landon M, Mabie W, et al. (2003) Randomized trial of inhaled beclomethasone dipropionate versus theophylline for moderate asthma during pregnancy. Am J Obstet Gynecol 190(3): 737-744.

20. Dombrowski MP, Schatz M, Wise R, Thom EA, Landon M, et al. (2004) Randomized trial of inhaled beclomethasone diproprianate versus theophylline for moderate asthma during pregnancy. Am J Obstet Gynecol 109(3): 737-744.

21. Williams MA, Gelaye B, Qiu C, Fida N, Cripe SM, et al. (2011) Habitual snoring and asthma comorbidity among pregnancy women. J Asthma 48(1): 91-97.

22. Sawicki E, Stewart K, Wong S, Leung L, Paul E, et al. (2011) Medication use for chronic health conditions by pregnant women attending an Australian maternity hospital. Aust NZ J Obstet Gynaecol 51(4): 333-338.

23. Sarkar M, Koren G, Kalra S, Ying A, Smorlesi C, et al. (2009) Montelukast use during pregnancy: a multicentre, prospective, comparative study of infant outcomes. Eur J Clin Pharmacol 65(12): 1259-1264.

24. Thompson MG, Kwong JC, Regan AK, Katz MA, Drews SJ, et al. (2019) Influenza Vaccine Effectiveness in Preventing Influenza-Associated Hospitalizations during 
Pregnancy: A Multi-Country Retrospective Test Negative Design Study, 2010-2016. Clin Infect Dis 68(9): 14441453.
25. Madhi SA, Cutland CL, Kuwanda L, Weinberg A, Hugo A, et al. (2014) Influenza Vaccination of Pregnant Women and Protection of Their Infants. N Engl J Med 371: 918931.

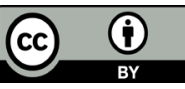

